\title{
Is Lipoprotein (a) a Risk Factor for Coronary Artery Ectasia?
}

\author{
Hanna K. Al-Makhamreh a, c, Ala' E. Shaban ${ }^{a}$, Sofia S. AlHaddadin ${ }^{a}$, Abedallatif A. AlSharif ${ }^{b}$, \\ Ruba A. Ghalaynia, Lana F. Daoud ${ }^{\text {a }}$, Baha M. Alshraideh ${ }^{\mathrm{a}}$
}

\begin{abstract}
Background: The pathophysiology of coronary artery ectasia (CAE) is under investigated and not well understood. Atherosclerosis is considered as the main etiologic factor for CAE in adults where more than $50 \%$ of CAE patients have atherosclerosis. Recently, lipoprotein (a) (Lp(a)) has emerged as a powerful risk factor for atherosclerosis and coronary artery disease (CAD). Serum levels of $\mathrm{Lp}(\mathrm{a})$ in patients with CAE have not been investigated. We assumed that $\mathrm{Lp}$ (a) may play a role in the pathogenesis of CAE. Principally, our study aims to identify whether $\mathrm{Lp}(\mathrm{a})$ is an independent risk factor for CAE.
\end{abstract}

Methods: Our study is a prospective pilot study. Study population was collected prospectively from pool of patients referred for elective cardiac catheterization at Jordan University Hospital (JUH) in the period extending from February 17, 2018 to June 31, 2018. Patients were referred for elective coronary angiography after being interviewed and physically examined by a cardiologist (HA). Patients with known history of CAD or who are already on anti-lipidemic drugs either documented in the medical records or by interviewing patients for history of revascularization were excluded from the study.

Results: Regarding the primary outcome, there was no significant difference in $\mathrm{Lp}$ (a) concentrations between normal and ectasia groups in the general sample (median: $17.5 \mathrm{mg} / \mathrm{dL}$ vs. $20.4 \mathrm{mg} / \mathrm{dL}$, P value $=0.38$ ).

Conclusions: Our study concludes that there is no detected relationship between elevated Lp(a) levels and developing CAE. CAE was more common in patients with low high-density lipoprotein (HDL) level (compared with patients with normal coronaries), higher total cholesterol level (compared with patients with non-obstructive stenosis) and higher hemoglobin A1c (HbA1c).

Keywords: Coronary ectasia; Coronary artery disease; Lipoprotein (a)

\footnotetext{
Manuscript submitted November 24, 2019, accepted December 30, 2019

${ }^{a}$ Cardiology Department, University of Jordan, Amman, Jordan ${ }^{b}$ Radiology Department, University of Jordan, Amman, Jordan ${ }^{c}$ Corresponding Author: Hanna K. Al-Makhamreh, Cardiology Department, University of Jordan, Amman, Jordan. Email: hmakhamreh@hotmail.com
}

doi: https://doi.org/10.14740/cr992

\section{Introduction}

Coronary artery ectasia (CAE) is defined as inappropriate dilatation of coronary artery exceeding the largest diameter of an adjacent normal vessel more than 1.5 fold $[1,2]$. CAE incidence is reported between $0.3 \%$ and $4.9 \%$ in patients undergoing coronary angiography [3]; these numbers are expected to rise with the increasing use of non-invasive coronary artery imaging such as computed tomography and magnetic resonance imaging. Clinically; patients with CAE with or without coronary artery narrowing may present with angina pectoris, positive stress test or acute coronary syndromes. Natural history of CAE and its management are not well established [3].

The pathophysiology of CAE is under investigated and not well understood. Atherosclerosis is considered as the main etiologic factor for CAE in adults where more than $50 \%$ of CAE patients have atherosclerosis. The exact mechanism of luminal dilatation in some vessels with atherosclerosis is unclear; CAE may be considered as exaggerated positive remodeling mechanism with the aim to preserve luminal size $[3,4]$. This remodeling is thought to be due to excessive degradation of the extracellular matrix by matrix metalloproteinases (MMPs) and other lytic enzymes in addition to thinning of tunica media due to severe chronic inflammation; actually no evidence of ectasia was observed in patients with intact media layer [3].

Risk factors for CAE are not well defined as those for stenotic atherosclerosis. Correlation of CAE with hypertension [5], diabetes mellitus (DM), dyslipidemia, smoking and family history of coronary artery disease is still controversial in literature [3, 4]. In addition, it has been reported that CAE may coexist with aneurysms of other arterial beds particularly abdominal aorta [6]. DM may even have a paradoxical role in the incidence of CAE. Although DM is a major risk factor for atherosclerosis; patients with DM were shown to have lower incidence of developing CAE, the hypothesis behind this paradox is that DM causes downregulation of MMP hence preventing exaggerated positive remodeling [7].

Recently, lipoprotein (a) (Lp(a)) has emerged as a powerful risk factor for atherosclerosis and coronary artery disease [8]. $\mathrm{Lp}(\mathrm{a})$ is a low density lipoprotein-like particle with the addition of apolipoprotein A (apoA) $[8,9]$. Lp(a) accumulates in blood vessel wall and inhibits binding of plasminogen to cell surface [8]. Lp(a) was evaluated in the literature to have gender differences in patients with proven coronary artery disease. There are differences in the studies' results; $L p(a)$ levels were believed to be higher in females with CAD than in males with 
CAD [10], but a more recent study found it higher in males than in females with CAD [11]. More recently, elevated serum Lp(a) levels have been demonstrated in patients with abdominal aortic aneurysms independently of atherosclerosis extent or risk factors [12]. Elevated Lp(a) levels have been also found in patients with intracranial aneurysms without significant atheromatous disease [13]. Elevated Lp(a) levels may cause endothelial and intimal damage with subsequent aneurysmal formation. In theory, aneurysmal dilatation of vessels and CAE may share same pathophysiology. Serum levels of Lp(a) in patients with CAE have not been investigated. We assumed that $\mathrm{Lp}$ (a) may play a role in the pathogenesis of CAE. Principally, our study aims to identify whether $\mathrm{Lp}(\mathrm{a})$ is an independent risk factor for CAE. Correlation between CAE and other controversial risk factors such as DM, lipid profile, hypertension and smoking will also be investigated. In addition, correlation of CAE with gender, and Lp(a) levels with gender will be investigated.

\section{Materials and Methods}

\section{Study design and population}

Our study is a prospective pilot study. Study population was collected prospectively from pool of patients referred for elective cardiac catheterization at Jordan University Hospital (JUH) in the period extending from February 17, 2018 to June 31, 2018. Patients were referred for elective coronary angiography after being interviewed and physically examined by a cardiologist (HA) due to a variety of reasons: stable angina and positive non-invasive evaluation by treadmill stress test or myocardial perfusion scintigraphy.

Patients were not enrolled if they presented with acute coronary syndrome. In addition patients already on anti-lipidemic drugs and patients with known history of coronary artery disease were excluded a priori from study.

Patients' age, gender and anthropometric data including height and weight for each patient were measured and registered at the time of enrollment.

\section{Laboratory tests}

Fasting antecubital venous blood samples were drawn for Lp(a), low-density lipoprotein (LDL), high-density lipoprotein (HDL), triglyceride, cholesterol, fasting blood sugar and hemoglobin A1c (HbAlc) at the catheterization lab prior to the catheterization. Samples were centrifuged immediately and stored at $-20{ }^{\circ} \mathrm{C}$. Lp(a) was measured by immunoturbidimetric assay in which human Lp(a) agglutinates with latex particles coated with anti-Lp(a) antibodies. The precipitate is determined turbidemetrically at $659 \mathrm{~nm}$. COBAS UNTEGRA systems were used for reagents handling; reagent was SR (latex particles coated with polyclonal anti-human Lp(a) antibodies (rabbit) glycine buffer: $170 \mathrm{mmol} / \mathrm{L}, \mathrm{pH} 7.3$; BSA, preservative). Measuring range was 7 - $240 \mathrm{nmol} / \mathrm{L}$; results were calculated in $\mathrm{nmol} / \mathrm{L}$ and then converted using the following conversion factor: $\mathrm{nmol} / \mathrm{L} \times 0.4167=\mathrm{mg} / \mathrm{dL}$.

\section{Cardiac catheterization}

Two cardiologists evaluated the results of coronary angiography and decided on patient's enrollment in each group, both cardiologists were blinded to reports of each other, when discrepancy was present a consensus was agreed upon reviewing patients' images.

\section{Primary and secondary outcomes}

The primary outcome of our study was to find if there is a correlation between $\mathrm{Lp}(\mathrm{a})$ levels and CAE in our sample population.

Secondary outcomes were to investigate if there are correlations between CAE and levels of $\mathrm{Lp}(\mathrm{a})$ in normolipidemic subjects, which was defined as patients with total triglycerides $<150 \mathrm{mg} / \mathrm{dL}$ and total cholesterol $<200 \mathrm{mg} / \mathrm{dL}$, and in borderline-lipid patients defined as patients with total triglycerides $<$ $200 \mathrm{mg} / \mathrm{dL}$ and total cholesterol $<240 \mathrm{mg} / \mathrm{dL}$; this was done to find if $\mathrm{Lp}(\mathrm{a})$ is an independent risk factor for CAE in patients with normal or borderline lipid levels. Other outcomes included evaluating if there is an association between CAE and other lipid profile parameters in the general sample: LDL, HDL, cholesterol, and total triglycerides in the general sample. Also, we aimed to find if there is any correlation between CAE and other variables such as age, gender, body mass index (BMI), hypertension, DM, and family history of CAD.

\section{Statistical analysis}

Data were tested for normality using Kolmogorov-Smirnov test. Categorical variables were presented as frequencies and percentages, normally distributed quantitative variables were expressed as means and standard deviations (SD), while nonnormally distributed quantitative variables were represented as median and interquartile range (IQR). Values of $\mathrm{Lp}(\mathrm{a})$ concentrations were compared according to CAD results by using the Mann-Whitney test to compare between two groups. A P value $<0.05$ was considered as significant. All the statistical analyses were performed using the SPSS software package (version 18.0, SPSS Inc. Chicago, Illinois).

\section{Ethical approval}

This study was approved by the Institutional Review Board (IRB) committee at the JUH. All procedures performed in studies involving human participants were in accordance with the ethical standards of the institutional research committee (the IRB; reference number: 69/2018), and with the 1964 Helsinki declaration and its later amendments or comparable ethical standards. This article does not contain any studies with animals performed by any of the authors. 
Table 1. Characteristics of the Patients

\begin{tabular}{ll}
\hline & Number (\%) \\
\hline Men & $58(63.7)$ \\
Smoking & $37(40.7)$ \\
Hypertension & $38(41.8)$ \\
Diabetic & $17(18.7)$ \\
Dyslipidemia & $10(11.0)$ \\
Family history & $11(12.1)$ \\
\hline
\end{tabular}

\section{Results}

A total of 512 patients were screened for eligibility; 91 patients met selection criteria. There were 58 males $(63.7 \%)$ and 33 females $(36.3 \%)$ (Table 1$)$. Mean age of the 91 patients was 55.6 \pm 1.3 (SD) years (Table 1). Age was normally distributed for the whole study population and for each separate study group. Mean age was $57.4 \pm 3.09$ years for ectasia group, $52.3 \pm 1.78$ years for patients with normal catheterization, $57.3 \pm 3.9$ years for patients with non-obstructive stenosis and $56.8 \pm 2.8$ years for patients with severe obstructive stenosis (Table 2).

Results of catheterization showed that, 35 (38.5\%) patients with normal catheterization, 15 (16.5\%) patients with ectasia, $18(19.8 \%)$ patients with non-obstructive stenosis and $23(25.3 \%)$ patients with severe obstructive stenosis.

$\mathrm{Lp}$ (a) values had a skewed distribution so they were represented as median and IQRs. Median level of $L p(a)$ in the general sample was $18.5 \mathrm{mg} / \mathrm{dL}$ (Fig. 1). Regarding the primary outcome, there was no significant difference in $\mathrm{Lp}(\mathrm{a})$ levels between normal and ectasia groups (median: 17.5 (9.8 - 32.3) mg/dL vs. 20.4 (12.3 - 32.8) mg/dL, respectively; P value $=0.38)($ Table 2$)$; also there was no significant difference in $\mathrm{Lp}$ (a) levels between any of the catheterization groups in study population. In the sample subgroup of patients with total triglycerides $<150 \mathrm{mg} / \mathrm{dL}$ and total cholesterol $<200 \mathrm{mg} / \mathrm{dL}$,

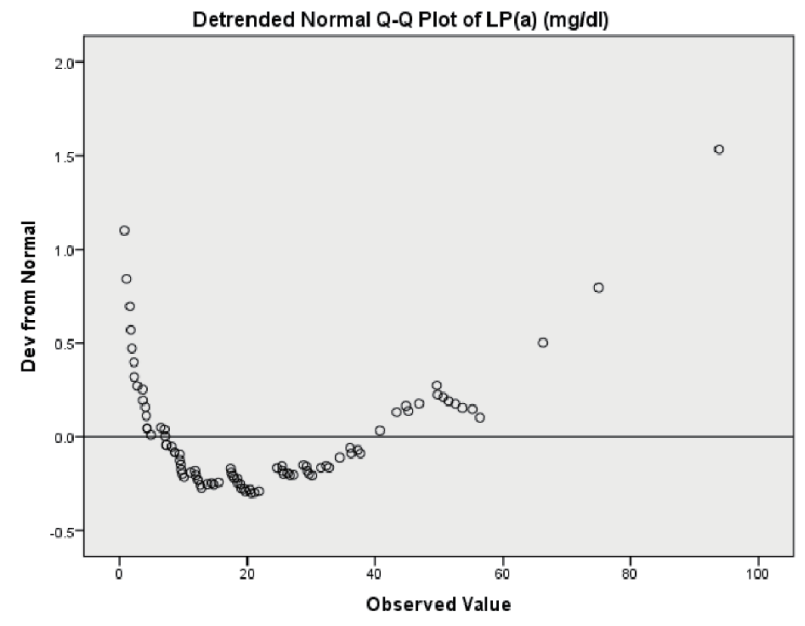

Figure 1. $L p(a)$ not normally distributed. $L p(a)$ values had a skewed distribution on Kolmogorov-Smirnov test; so they were represented as median and interquartile ranges. Median level of $L p(a)$ in the general sample was $18.5 \mathrm{mg} / \mathrm{dL}$. Lp(a): lipoprotein (a).

there were six patients with ectasia, 22 with normal coronaries, 13 with non-obstructive coronary artery disease and 13 with obstructive coronary artery disease; there was no significant difference in the levels of $\mathrm{Lp}(\mathrm{a})$ between ectasia patient and normal group patients ( $\mathrm{P}$ value $=0.54)$. In addition, in patients with total triglycerides $<200 \mathrm{mg} / \mathrm{dL}$ and total cholesterol $<$ $240 \mathrm{mg} / \mathrm{dL}$, there were nine patients with ectasia, 31 with normal coronaries, 14 with non-obstructive coronary artery disease and 15 with obstructive coronary artery disease ( $P$ value $=0.14$ ).

On Kolmogorov-Smirnov test for the 91 patients, LDL and total cholesterol were normally distributed while HDL and total triglyceride were not normally distributed. HDL levels were significantly lower in CAE group compared with normal coronaries group (median: $36 \mathrm{mg} / \mathrm{dL}(31.5$ - 47) vs. $47 \mathrm{mg} /$ $\mathrm{dL}(39$ - 57), respectively; P value $=0.02)$ (Table 2). For total

Table 2. Clinical Characteristics and Lipid Profile for Normal and Ectasia Groups

\begin{tabular}{|c|c|c|c|c|c|}
\hline Parameters & Normal $(n=35)$ & Ectasia $(n=15)$ & Non-obstructive CAD & Obstructive CAD & P value \\
\hline Age in years $($ mean $\pm \mathrm{SD})$ & $52.3 \pm 1.8$ & $57.4 \pm 3.1$ & $57.3 \pm 3.9$ & $56.8 \pm 2.8$ & 0.17 \\
\hline Weight $(\mathrm{kg})($ mean $\pm \mathrm{SD})$ & $86.4(2.84)$ & $94.9(5.4)$ & 86.8 & 81.4 & 0.22 \\
\hline BMI (kg/m²) (median (IQR)) & $29.9(28.4-33.1)$ & $31.6(27.6-36.2)$ & $29.7 \mathrm{~kg} / \mathrm{m}^{2}( \pm 1.6, \mathrm{SD})$ & $28.86( \pm 1.33, \mathrm{SD})$ & 0.32 \\
\hline Lp(a) (mg/dL) (median (IQR)) & $17.5(9.8-32.3)$ & $20.4(12.3-32.8)$ & & & 0.38 \\
\hline HDL (median (IQR)) & $47(39-57)$ & $36(31.5-47)$ & 38.00 & 39.00 & 0.02 \\
\hline Cholesterol $($ mean $\pm \mathrm{SD})$ & $170.0(6.2)$ & $187.6(9.9)$ & 156 & 169.4 & 0.16 \\
\hline Triglyceride (median (IQR)) & $102(71-138.5)$ & $110(83-186.5)$ & 101 & 90 & 0.19 \\
\hline Fasting blood sugar (median (IQR)) & $99(90.4-112.4)$ & $104.5(95-129.1)$ & 112 & 102 & 0.24 \\
\hline HbAlc (median (IQR)) & $5.7(5.4-6.1)$ & $5.9(5.7-7)$ & 5.8 & 5.5 & 0.02 \\
\hline
\end{tabular}

CAD: coronary artery disease; SD: standard deviations; IQR: interquartile range; BMI: body mass index; Lp(a): lipoprotein (a); LDL: low-density lipoprotein; HDL: high-density lipoprotein; HbA1c: hemoglobin A1c. 


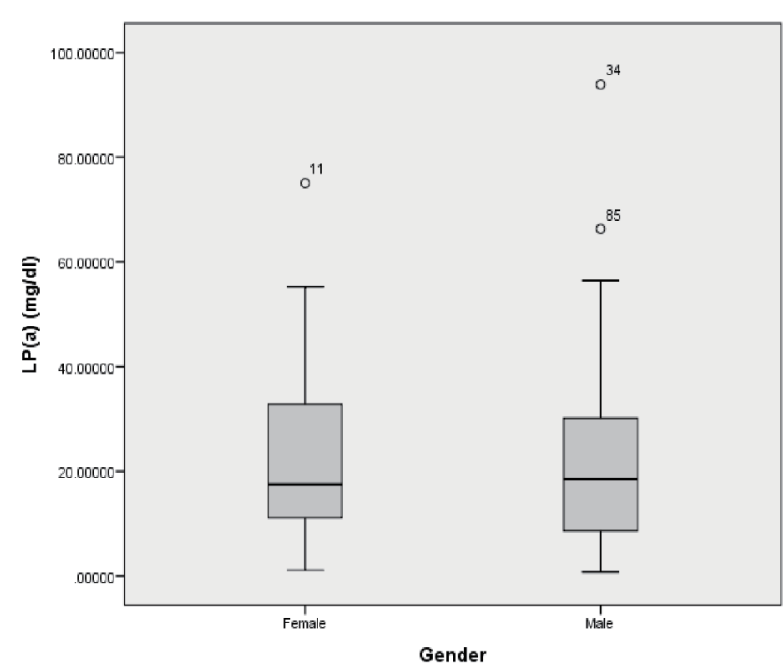

Figure 2. $L p(a)$ levels in males and females in the general sample. There is no significant difference in $L p(a)$ levels between males and females (median: $18.5 \mathrm{mg} / \mathrm{dL}$ in males vs. $17.5 \mathrm{mg} / \mathrm{dL}$ in females, $\mathrm{P}$ value $=0.811)$. $L p(a)$ : lipoprotein $(a)$.

cholesterol levels, the only observed significant difference was between ectasia group and non-obstructive stenosis group with values being higher in the ectasia group (median: $184.5 \mathrm{mg} / \mathrm{dL}$ vs. $159 \mathrm{mg} / \mathrm{dL}$, respectively, P value $=0.04)($ Table 2$)$.

There is a significant correlation between ectasia group and gender; males were 5.33 times more likely to have ectasia compared to females (odds ratio $=5.33,95 \%$ confidence inter$\operatorname{val}(1.3-22.3))$.

There was no significant difference in $L p(a)$ levels between males and females in the general sample (median: $18.5 \mathrm{mg} / \mathrm{dL}$ in males vs. $17.5 \mathrm{mg} / \mathrm{dL}$ in females, $\mathrm{P}$ value $=0.811)($ Fig. 2). In addition, no significant difference was found in $\mathrm{Lp}(\mathrm{a})$ levels between males and females in each separate catheterization group. In normal coronaries group, $\mathrm{Lp}(\mathrm{a})$ levels were 18 $\mathrm{mg} / \mathrm{dL}$ in males vs. $16.5 \mathrm{mg} / \mathrm{dL}$ in females, $\mathrm{P}$ value $=0.7$. In patients with non-obstructive stenosis, $\mathrm{Lp}$ (a) levels were 19 $\mathrm{mg} / \mathrm{dL}$ in males vs. $18.9 \mathrm{mg} / \mathrm{dL}$ in females, $\mathrm{P}$ value $=0.48$. In patients with obstructive stenosis, $\mathrm{Lp}$ (a) levels were $23.8 \mathrm{mg} /$ $\mathrm{dL}$ in males vs. $30.6 \mathrm{mg} / \mathrm{dL}$ in females, $\mathrm{P}$ value $=0.17$. Lastly, in patients with CAE, Lp(a) levels were 20.8 in males vs. 19 in females, $P$ value $=0.47$.

BMI was normally distributed for the whole study population and for each separate study group. Mean value of BMI in the general sample was $30.3 \pm 0.67(\mathrm{SD}) \mathrm{kg} / \mathrm{m}^{2}$. Mean BMI in ectasia group was $32.7 \pm 1.7$ (SD) $\mathrm{kg} / \mathrm{m}^{2}$, in normal group $30.7 \pm 1.02(\mathrm{SD}) \mathrm{kg} / \mathrm{m}^{2}$, non-obstructive stenosis group $29.7 \pm$ 1.6 (SD) $\mathrm{kg} / \mathrm{m}^{2}$ and in severe obstructive group $28.86 \pm 1.33$ (SD) $\mathrm{kg} / \mathrm{m}^{2}$ with no significant difference between any of the groups (Table 2).

In addition, there was no significant correlation between hypertension and CAE. On the other hand, HbAlc values were significantly higher in ectasia group compared to normal (5.9 $\mathrm{mg} / \mathrm{dL}$ vs. $5.7 \mathrm{mg} / \mathrm{dL}$, P value $=0.02)($ Table 2$)$.

In our study sample, $37(40.7 \%)$ of the patients were smokers (Table 1); five patients with ectasia in comparison to 14 in normal group. There was no significant correlation be- tween smoking and having ectasia.

In patients with ectasia, only one patient had a family history of coronary artery disease while there were 14 in normal group. On Pearson Chi-square correlation test, there was no significant correlation between ectasia and having a family history of coronary artery disease; $\mathrm{P}$ value $(0.086)$ is greater than the significance level (0.05).

\section{Discussion}

CAE is defined as $\geq 1.5$-fold dilation of a coronary artery with respect to the diameter of adjacent normal segments $[1,2,4]$. $\mathrm{CAE}$ which is considered to be a variant of atherosclerotic diseases is uncommonly encountered in diagnostic angiographic procedures with an incidence of $0.3-4.9 \%$ [3]. Exaggerated expansive remodeling as a result of enzymatic degradation and media thinning causing a chronic inflammation is thought to participate in the etiology of CAE $[3,4]$.

$\mathrm{Lp}(\mathrm{a})$ is one of the lipoproteins which is similar in structure to LDL except that $\mathrm{Lp}(\mathrm{a})$ has apoA bound to apoB-100 via one disulfide bond $[8,9]$. $L p(a)$ was proved to be an independent causal factor of atherosclerotic diseases; levels above $(20-30 \mathrm{mg} / \mathrm{dL}$ ) are associated with 2-fold risks of developing coronary artery disease [9]. In other studies, elevated Lp(a) levels were shown to be associated with abdominal aortic aneurysms (AAA) which resembles the pathophysiology of CAE $[12,14,15]$. To our knowledge, the relationship between CAE and $\mathrm{Lp}(\mathrm{a})$ was never studied in current medical literature.

Our study sample enrolled patients being admitted for elective cardiac catheterization due to variable cardiac indications. Patients were not enrolled in the study in the setting of acute coronary syndrome as previous studies showed altered lipids levels in these patients; it was reported that multiple lipids such as LDL, HDL and total cholesterol decrease following acute coronary syndrome while triglyceride level increased $[16,17]$. Furthermore, our study excluded a priori patients with previous history of coronary artery disease and patients on anti-lipidemic drugs to assure that lipid levels including $\mathrm{Lp}$ (a) are not altered by the use of medications.

We found there was no significant difference in $\operatorname{Lp}(a)$ levels between the different catheterization categories in the whole sample; $\mathrm{Lp}(\mathrm{a})$ levels in normal and ectasia groups were (median: $17.5(9.8$ - 32.3) mg/dL vs. $20.4(12.3-32.8) \mathrm{mg} /$ $\mathrm{dL}, \mathrm{P}$ value $=0.38$ ). To our knowledge this is the first study to evaluate possible correlations of $\mathrm{Lp}$ (a) levels with CAE. However, contrary to our expectations, no correlations were found.

We also found no significant correlation between $\operatorname{Lp}(\mathrm{a})$ levels and CAE in the normolipidemic to borderline lipidemic patients (triglycerides $<150$ and total cholesterol $<200$ or triglycerides $<200$ and total cholesterol $<240$ ). The role of elevated Lp(a) in the pathophysiology of CAE in patients with normal to borderline levels of lipids cannot be therefore confirmed.

$\mathrm{Lp}$ (a) accumulates in blood vessel wall inhibiting binding of plasminogen to cell surface. It should be noted here that a previous study of Sofi et al showed significantly increased levels of plasminogen activator inhibitor-1 and $L p(a)$ in patients with abdominal aortic aneurysms [18]. In addition the 
study of Cicek et al demonstrated significantly increased levels of plasminogen activator inhibitor-1 in patients with isolated CAE compared to the normal coronary patients [19]. Later another study supported these finding showing decreased levels of plasminogen activity and increased concentrations of plasmin inhibitors in CAE patients [20]. Further studies are needed to investigate association between $\mathrm{Lp}(\mathrm{a})$ and plasminogen in patients with CAE.

In regards to gender, our study showed that males were 5.33 times more likely (odds ratio $=5.33,95 \%$ confidence interval (1.28 - 22.32)) to develop ectasia more than females. This is higher than the gender trend reported in literature with male to female ration of $3: 1$, this higher ratio is mostly due to more males recruited in this study (63\% of study population) [3].

There was, however, no significant difference in $\mathrm{Lp}(\mathrm{a})$ levels between males and females in the general sample (median: $18.5 \mathrm{mg} / \mathrm{dL}$ in males vs. $17.5 \mathrm{mg} / \mathrm{dL}$ in females, $\mathrm{P}$ value $=0.81)$; neither there was any significant difference in $\mathrm{Lp}(\mathrm{a})$ levels between males and females in any of the catheterization groups; ectasia, normal coronaries, non-obstructive stenosis, and obstructive stenosis.

Being a part of the spectrum of atherosclerotic disease, ectasia was shown to be associated with many atherosclerotic risks including dyslipidemia such as lower HDL levels compared with the non-ectasia patients in the study of Sudhir et al [21]; levels in that study were $47.2 \pm 2.6 \mathrm{mg} / \mathrm{dL} ; 55.8 \pm 1.2$ $\mathrm{mg} / \mathrm{dL}$, respectively $(\mathrm{P}$ value $=0.003)$. Our study confirmed this association; and we found that lower HDL levels in ectasia group compared with the normal coronary patients; median: $36(31.5$ - 47) mg/dL vs. $47(39-57) \mathrm{mg} / \mathrm{dL}$, respectively $(\mathrm{P}$ value $=0.02)$. Familial hypercholesterolemia was also shown to be associated with ectasia [21], total cholesterol levels in our study were significantly higher in the ectasia group compared with the non-obstructive coronary stenosis group; mean: $187.65 \mathrm{mg} / \mathrm{dL}$ vs. $156.0 \mathrm{mg} / \mathrm{dL}$, respectively $(\mathrm{P}$ value $=0.04)$.

There was a controversy in literature regarding the association of hypertension, DM, smoking and family history with CAE. Our study showed a significant correlation between CAE and elevated $\mathrm{HbA} 1 \mathrm{c}$ levels as compared to the normal coronary group $(5.9 \mathrm{mg} / \mathrm{dL}$ vs. $5.7 \mathrm{mg} / \mathrm{dL}, \mathrm{P}$ value $=0.021)$. In addition, we found a significant correlation between family history of coronary artery disease and CAE. However, there was no significant association of hypertension or smoking with ectasia.

\section{Acknowledgments}

None to declare.

\section{Financial Disclosure}

This study did not receive any fund from any party.

\section{Conflict of Interest}

The authors declare that they have no conflict of interest.

\section{Informed Consent}

Informed consent was obtained from all individual participant included in the study.

\section{Author Contributions}

Hanna K. Al-Makhamreh contributed to formulation of the study idea, literature review, data collection (recruiting patients for entry and performing the catheterization), data analysis, and thesis writing. Ala' E. Shaban contributed to literature review, data collection (exclusion of patients based on exclusion criteria, getting blood samples and data entry), data analysis, and thesis writing. Sofia AlHaddadin contributed to literature review, data collection (exclusion of patients based on exclusion criteria, getting blood samples and data entry), and thesis writing. Abdellatif A. Alsharif involved in data analysis and proof reading of the thesis. Ruba A. Ghalayni involved in data collection (exclusion of patients based on exclusion criteria, getting blood samples and data entry). Lana F. Daoud contributed to data collection (exclusion of patients based on exclusion criteria, getting blood samples and double checking for data entry). Baha M. Alshraideh involved in data collection (exclusion of patients based on exclusion criteria, getting blood samples and data entry).

\section{References}

1. Pinar Bermudez E, Lopez Palop R, Lozano MartinezLuengas I, Cortes Sanchez R, Carrillo Saez P, Rodriguez Carreras R, Pico Aracil F, et al. [Coronary ectasia: prevalence, and clinical and angiographic characteristics]. Rev Esp Cardiol. 2003;56(5):473-479.

2. Nyamu P, Ajit MS, Joseph PK, Venkitachalam L, Sugirtham NA. The prevalence and clinical profile of angiographic coronary ectasia. Asian Cardiovasc Thorac Ann. 2003;11(2):122-126.

3. Ulas Ozcan O, Gulec S. Coronary artery ectasia. Cor et Vasa. 2013;55(3):e242-e247.

4. Devabhaktuni S, Mercedes A, Diep J, Ahsan C. Coronary artery ectasia-a review of current literature. Curr Cardiol Rev. 2016;12(4):318-323.

5. Swaye PS, Fisher LD, Litwin P, Vignola PA, Judkins MP, Kemp HG, Mudd JG, et al. Aneurysmal coronary artery disease. Circulation. 1983;67(1):134-138.

6. Baugh M, Gavrilovic J, Davies I, Hughes D, Sampson M. Cardiovascular Diabetology. 2003;2(1):3.

7. Kornowski R, Mintz GS, Lansky AJ, Hong MK, Kent KM, Pichard AD, Satler LF, et al. Paradoxic decreases in atherosclerotic plaque mass in insulin-treated diabetic patients. Am J Cardiol. 1998;81(11):1298-1304.

8. Nordestgaard BG, Langsted A. Lipoprotein (a) as a cause of cardiovascular disease: insights from epidemiology, genetics, and biology. J Lipid Res. 2016;57(11):1953-1975.

9. Maranhao RC, Carvalho PO, Strunz CC, Pileggi F. Lipoprotein (a): structure, pathophysiology and clinical impli- 
cations. Arq Bras Cardiol. 2014;103(1):76-84.

10. Frohlich J, Dobiasova M, Adler L, Francis M. Gender differences in plasma levels of lipoprotein (a) in patients with angiographically proven coronary artery disease. Physiol Res. 2004;53(5):481-486.

11. Malek F, Dvorak J, Skalnikova V, Mates M, Kmonicek P, Vavrova Z, Neuzil P. Correlation of lipoprotein(a) with the extent of coronary artery disease in patients with established coronary atherosclerosis: gender differences. Eur J Prev Cardiol. 2015;22(5):603-605.

12. Kubota Y, Folsom AR, Ballantyne CM, Tang W. Lipoprotein(a) and abdominal aortic aneurysm risk: The Atherosclerosis Risk in Communities study. Atherosclerosis. 2018;268:63-67.

13. Phillips J, Roberts G, Bolger C, el Baghdady A, BouchierHayes D, Farrell M, Collins P. Lipoprotein (a): a potential biological marker for unruptured intracranial aneurysms. Neurosurgery. 1997;40(5):1112-1115; discussion 11151117.

14. Befeler B, Aranda MJ, Embi A, Mullin FL, El-Sherif N, Lazzara R. Coronary artery aneurysms: study of the etiology, clinical course and effect on left ventricular function and prognosis. Am J Med. 1977;62(4):597-607.

15. Stajduhar KC, Laird JR, Rogan KM, Wortham DC. Coronary arterial ectasia: increased prevalence in patients with abdominal aortic aneurysm as compared to occlusive atherosclerotic peripheral vascular disease. Am Heart J. 1993;125(1):86-92.

16. Rosenson RS, Brewer HB, Rader DJ. Lipoproteins as biomarkers and therapeutic targets in the setting of acute coronary syndrome. Circ Res. 2014;114(12):1880-1889.

17. Balci B. The modification of serum lipids after acute coronary syndrome and importance in clinical practice. Curr Cardiol Rev. 2011;7(4):272-276.

18. Sofi F, Marcucci R, Giusti B, Pratesi G, Lari B, Sestini I, Lo Sapio P, et al. High levels of homocysteine, lipoprotein (a) and plasminogen activator inhibitor-1 are present in patients with abdominal aortic aneurysm. Thromb Haemost. 2005;94(5):1094-1098.

19. Cicek Y, Durakoglugil ME, Erdogan T, Yilmaz A, Uydu HA, Saglam H, Cetin M, et al. Increased plasminogen activator inhibitor-1 levels in patients with isolated coronary artery ectasia. J Thromb Thrombolysis. 2012;33(1):120123.

20. Wu W, Liu R, Chen L, Chen H, Zhang S. Disequilibrium of blood coagulation and fibrinolytic system in patients with coronary artery ectasia. Medicine (Baltimore). 2016;95(8):e2779.

21. Sudhir K, Ports TA, Amidon TM, Goldberger JJ, Bhushan $\mathrm{V}$, Kane JP, Yock P, et al. Increased prevalence of coronary ectasia in heterozygous familial hypercholesterolemia. Circulation. 1995;91(5):1375-1380. 